\title{
Mostow and Artin Awarded 2013 Wolf Prize
}

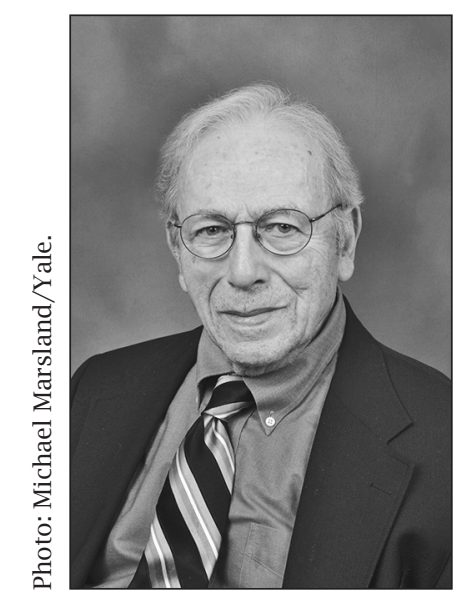

George D. Mostow

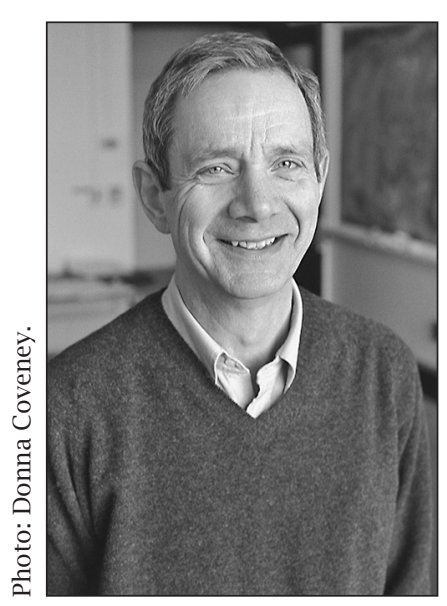

Michael Artin
The 2013 Wolf Prize in Mathematics has been awarded to:

GEORGE D. Mostow, Yale University, "for his fundamental and pioneering contribution to geometry and Lie group theory."

MiCHAEL ARTIN, Massachusetts Institute of Technology, "for his fundamental contributions to algebraic geometry, both commutative and noncommutative."

The prize of US $\$ 100,000$ will be divided equally between the prizewinners. The list of previous recipients of the Wolf Prize in Mathematics is available on the website of the Wolf Foundation, http: //www. wolffund.org.i1.

\section{Description of the Prizewinners' Work}

The following descriptions of the prizewinners' work were prepared by the Wolf Foundation.

George D. Mostow has made a fundamental and pioneering contribution to geometry and Lie group theory. His most celebrated accomplishment in these fields is the discovery of the completely new rigidity phenomenon in geometry, the Strong Rigidity Theorems. These theorems are some of the greatest achievements in mathematics in the second half of the twentieth century. This established a deep connection between continuous and discrete groups or, equivalently, a remarkable connection between topology and geometry. Mostow's

DOI: http://dx.doi.org/10.1090/noti990 rigidity methods and techniques opened a floodgate of investigations and results in many related areas of mathematics. Mostow's emphasis on the "action at infinity" has been developed by many mathematicians in a variety of directions. It had a huge impact in geometric group theory, in the study of Kleinian groups and of low-dimensional topology, and in work connecting ergodic theory and Lie groups. Mostow's contribution to mathematics is not limited to strong rigidity theorems. His work on Lie groups and their discrete subgroups, which was done during 1948-1965, was very influential. Mostow's work on examples of nonarithmetic lattices in two- and three-dimensional complex hyperbolic spaces (partially in collaboration with P. Deligne) is brilliant and led to many important developments in mathematics. In Mostow's work one finds a stunning display of a variety of mathematical disciplines. Few mathematicians can compete with the breadth, depth, and originality of his works.

Michael Artin is one of the main architects of modern algebraic geometry. His fundamental contributions encompass a bewildering number of areas in this field.

To begin with, the theory of étale cohomology was introduced by Michael Artin jointly with Alexander Grothendieck. Their vision resulted in the creation of one of the essential tools of modern algebraic geometry. Using étale cohomology, Artin showed that the finiteness of the Brauer group of a surface fibered by curves is equivalent to the Birch and Swinnerton-Dyer conjecture for the Jacobian of a general fiber. In a very original paper Artin and Swinnerton-Dyer proved the conjecture for an elliptic $K 3$ surface.

He also collaborated with Barry Mazur to define étale homotopy, another important tool in algebraic geometry, and more generally to apply ideas from algebraic geometry to the study of diffeomorphisms of compact manifolds.

We owe to Michael Artin, in large part, also the introduction of algebraic spaces and algebraic stacks. These objects form the correct category in which to perform most algebro-geometrical constructions, and this category is ubiquitous in the theory of moduli and in modern intersection theory. Artin discovered a simple set of conditions for a functor to be represented by an algebraic 
space. His "approximation theorem" and his "existence theorem" are the starting points of the modern study of moduli problems. Artin's contributions to the theory of surface singularities are of fundamental importance. In this theory he introduced several concepts that immediately became seminal to the field, such as the concepts of rational singularity and of fundamental cycles.

In yet another example of the sheer originality of his thinking, Artin broadened his reach to lay rigorous foundations to deformation theory. This is one of the main tools of classical algebraic geometry, which is the basis of the local theory of moduli of algebraic varieties.

Finally, his contribution to noncommutative algebra has been enormous. The entire subject changed after Artin's introduction of algebrogeometrical methods in this field. His characterization of Azumaya algebras in terms of polynomial identities, which is the content of the Artin-Procesi theorem, is one of the cornerstones in noncommutative algebra. The Artin-Stafford theorem, stating that every integral projective curve is commutative, is one of the most important achievements in noncommutative algebraic geometry.

Artin's mathematical accomplishments are astonishing for their depth and their scope. He is one of the great geometers of the twentieth century.

\section{Biographical Sketches}

George Mostow received his Ph.D. from Harvard University in 1948. He has served on the faculties of Johns Hopkins University (1952-1961) and Yale University (1961-1999). He is a member of the National Academy of Sciences (elected 1974) and has served as the president of the American Mathematical Society (1987-1988). He was awarded the 1993 Leroy P. Steele Prize for Seminal Contribution to Research for his book Strong Rigidity of Locally Symmetric Spaces (1973).

Michael Artin was born in Hamburg, Germany, in 1934, son of mathematician Emil Artin. He received his Ph.D. from Harvard University in 1960. He has been affiliated with the Institut des Hautes Études Scientifiques, as well as with MIT, whose faculty he joined in 1963. He was awarded the Steele Prize for Lifetime Achievement in 2002. In 2005 he received the Harvard Centennial Medal. He served as president of the AMS in 1991-1992. He is a member of the National Academy of Sciences and a fellow of the American Academy of Arts and Sciences, the American Association for the Advancement of Science, the Society for Industrial and Applied Mathematics, and the American Mathematical Society.

Israeli President Shimon Peres will present the prizes in May at a special session hosted by the Knesset.

\section{Daubechies and Mumford Receive BBVA Foundation Award}

INGRID DAUBECHIES of Duke University and DAVID MUMFORD of Brown University have been honored with the BBVA Foundation Frontiers of Knowledge Award in the Basic Sciences category for their work in pure mathematics, which, according to the prize citation, has "strongly influenced diverse fields of application, ranging from data compression to pattern recognition." Both researchers have formulated solutions to varied and complex problems starting from the vantage point of pure mathematics but guided by a multidisciplinary approach. The award carries a cash prize of 400,000 euros (approximately US\$550,000).

The jury singled out Daubechies, who trained as a physicist, for her work on "wavelets, which resulted in a new approach to data compression, with

DOI: http://dx.doi.org/10.1090/noti986 a strong impact on a multitude of technologies, including efficient audio and video transmission and medical imaging." Mumford's mathematical research has had considerable influence among theoretical physicists. He was recognized particularly for "his contributions to algebraic geometry and to the mathematics of computer vision. He has applied tools of variational calculus to the theory of vision and developed statistical models for imaging and pattern recognition. His work has had a lasting impact in both pure and applied mathematics."

A leader in the field of algebraic geometry, Mumford turned in the 1980s to a new problem: how to mathematically render the human ability to understand an image. One of his insights was that the brain operates by combining previous knowledge with what it is perceiving right now. His 